\title{
Delayed umbilical cord clamping effects on caesarean delivery neonates under general anaesthesia: A prospective cohort study
}

\author{
qian hu ${ }^{1}$, Rui Zhong ${ }^{2}$, Peng Bai ${ }^{3}, \mathrm{Xue}_{\mathrm{Xiao}}{ }^{3}$, Bin Zhou ${ }^{3}$, Teng $\mathrm{He}^{3}$, Lin Zhang ${ }^{3}$, and \\ Huafeng $\mathrm{Li}^{4}$ \\ ${ }^{1}$ Sichuan University West China Second University Hospital \\ ${ }^{2}$ Huazhong University of Science and Technology Union Shenzhen Hospital \\ ${ }^{3}$ Affiliation not available \\ ${ }^{4}$ Sichuan University
}

November 16, 2020

\begin{abstract}
Objective: To investigate the effect of delayed umbilical cord clamping on neonatal outcomes following caesarean delivery under general anaesthesia. Design: Prospective cohort study. Setting: West China Second University Hospital Sample: Neonates born by caesarean delivery under general anaesthesia after 35 gestational weeks. Methods: Neonates were assigned to Groups A or B if they received early or delayed cord clamping, respectively. Main Outcome Measures: Umbilical arterial blood gas analysis indicators, Apgar scores, resuscitation procedure incidence, peak bilirubin, and neonatal morbidity were compared between the two groups. Results: Group A had 29 and Group B had 21 participants. There were no significant differences in any of the outcome measures between the two groups. We classified five periods during caesarean delivery: aesthetic induction (Period 1), skin incision (Period 2), myometrium incision (Period 3), delivery of the neonate (Period 4), and time of cord clamping (Period 5). One-minute Apgar scores were negatively correlated with cord-clamping time $(\mathrm{r}=-0.426, \mathrm{P}=0.002)$. Peak bilirubin value was correlated with Periods 2,3 , and 5 ( $\mathrm{r}=0.347, \mathrm{P}=0.014 ; \mathrm{r}=0.411, \mathrm{P}=0.003 ; \mathrm{r}=-0.289, \mathrm{P}=0.042$, respectively). The remaining secondary outcomes were not correlated with any of the five periods. The peak bilirubin value was $(9.712+0.006$ $\times$ Period $2+0.006 \times$ Period 3-0.026 $\times$ Period 5) $(\mathrm{R} 2=0.313)$. Conclusions: In caesarean delivery under general anaesthesia, delayed cord clamping within a certain period may partially prolong the duration of neonatal exposure to general anaesthesia drugs. However, delayed cord clamping is a safe and feasible technique for clinical application.
\end{abstract}

\section{Hosted file}

covering_letter final edition.pdf available at https://authorea.com/users/376146/articles/ 493215-delayed-umbilical-cord-clamping-effects-on-caesarean-delivery-neonates-undergeneral-anaesthesia-a-prospective-cohort-study

\section{Hosted file}

manuscript_final.pdf available at https://authorea.com/users/376146/articles/493215delayed-umbilical-cord-clamping-effects-on-caesarean-delivery-neonates-under-generalanaesthesia-a-prospective-cohort-study

\section{Hosted file}

supplementary_materials.pdf available at https://authorea.com/users/376146/articles/493215delayed-umbilical-cord-clamping-effects-on-caesarean-delivery-neonates-under-generalanaesthesia-a-prospective-cohort-study 

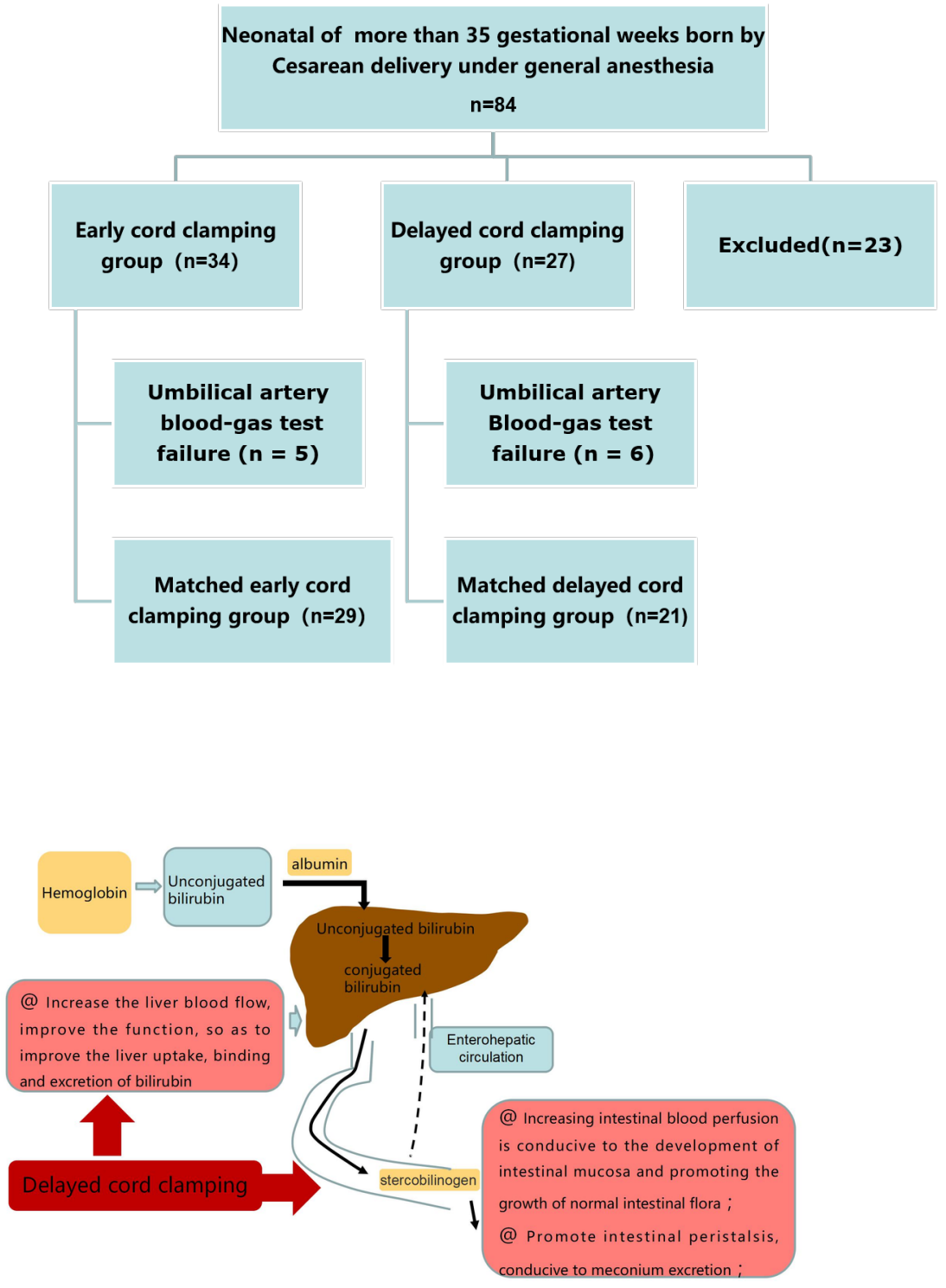